\title{
Modeling Electron Fractionalization with Unconventional Fock Spaces
}

\author{
Emilio Cobanera ${ }^{a}$ \\ Institute for Theoretical Physics, Center for Extreme Matter and Emergent Phenomena, \\ Utrecht University, Leuvenlaan 4, 3584 CE Utrecht, The Netherlands
}

\begin{abstract}
It is shown that certain fractionally-charged quasiparticles can be modeled on $D$-dimensional lattices in terms of unconventional yet simple Fock algebras of creation and annihilation operators. These unconventional Fock algebras are derived from the usual fermionic algebra by taking roots (the square root, cubic root, etc.) of the usual fermionic creation and annihilation operators. If the fermions carry non-Abelian charges, then this approach fractionalizes the Abelian charges only. In particular, the $m$ th-root of a spinful fermion carries charge $e / m$ and spin $1 / 2$. Just like taking a root of a complex number, taking a root of a fermion yields a mildly non-unique result. As a consequence, there are several possible choices of quantum exchange statistics for fermionroot quasiparticles. These choices are tied to the dimensionality $D=1,2,3, \ldots$ of the lattice by basic physical considerations. One particular family of fermion-root quasiparticles is directly connected to the parafermion zero-energy modes expected to emerge in certain mesoscopic devices involving fractional quantum Hall states. Hence, as an application of potential mesoscopic interest, I investigate numerically the hybridization of Majorana and parafermion zero-energy edge modes caused by fractionalizing but charge-conserving tunneling.
\end{abstract}

\footnotetext{
a Present address: Department of Physics and Astronomy, Dartmouth College, 6127 Wilder Laboratory, Hanover, NH 03755, USA
} 


\section{INTRODUCTION}

Lattice-regularized theories are everywhere in physics, because they capture in a transparent way the interplay between internal symmetries, strong correlations [1], topological quantum orders [2-4], dualities [5, 6], and disorder [7, 8]. They represent a rich class of models naturally suited for numerical simulations, notwithstanding the fact that they pose exponentially hard computational problems more often than not. Quantum information processing tools are best adapted to lattice-regularized systems [9]. Because microscopically motivated lattice theories can be extraordinarily detailed, it is often necessary or desirable to search for simplifications. The schematic models that result, sometimes derived and sometimes conjectured, are regarded as effective, and one seeks to incorporate in them as much as one believes to know about the nature of the quasiparticle excitations dominant in the energy scales of interest.

This paper focuses on one particular topic where effective lattice models have played a relatively small role thus far: electron fractionalization. Consider for concreteness the SuSchrieffer-Heeger (SSH) model [10] of electrons coupled to phonons in one dimension. In this model of the linear polyacetylene molecule, kinks in the phonon field carry a fractional amount of electric charge, due to dressing by the electronic degrees of freedom. Typically, this dressing mechanism is investigated in an approximation in which the kinks are static.

This approximation is very fruitful and admits grand generalizations in field theory [11]. But in a less simplified picture where the phonons are not frozen, the electrons might coexist with dynamic kink excitations, and channels for electron breakup into fractionally charged kinks and recombination might perhaps exist. If this were the case, an effective lattice field theory of such a regime, especially a simple one, would be quite different from the starting point, the SSH model. Presumably it would feature effective creation and annihilation operators of dressed kinks. But what should be the algebra of such effective creation and annihilation operators?

While the SSH model is arguably the simplest model of charge fractionalization, the idea of dynamic, fractionally charged quasiparticles has been pursued much further in the context of fractional quantum Hall $(\mathrm{FQH})$ effects $[12,13]$. In these systems, the exact microscopic origin of fractionally-charged quasiparticles is still an active area of research [14]. However, for the FQH states characterized by Jain fractions $\nu=p / q$ ( $q$ must be an 
odd integer), there is agreement on the nature of these quasiparticles on three basic points. First, the charge of the elementary [15] quasiparticles is $e^{*}=e / q$, independently of $p(e<0$ is the charge of the electron). Second, these quasiparticles display unconventional exchange quantum statistics characterized by the statistical angle $\theta=e^{i \pi / q}[16]$. Third, an electron entering a FQH fluid may undergo charge breakup, splitting into several mobile, if it were not for disorder, quasiparticles, and, conversely, these quasiparticles may recombine into an electron [17]. The second point, unconventional quantum statistics, is hard to probe experimentally. Shot noise experiments are good evidence for the first and third points [18]. One can summarize these three basic ideas by saying that the electron is fractionalized in the FQH liquids associated to the Jain fractions, in the very precise sense that it breaks up into indistinguishable quasiparticles that behave as fractions of the original electron.

In this paper it will be shows that it is possible to capture the three basic physical features of a "fraction of an electron" in terms of unconventional Fock algebras of creation and annihilation operators. These unconventional Fock operators create/annihilate a fraction of a fermion in a surprisingly literal sense, and can be used for describing a fraction of a spinful fermion and, more generally, a fraction of a fermion carrying any sort of nonAbelian internal symmetry. The unconventional Fock algebras of this paper can be chosen to display anyonic features, but are not mathematically tied to space dimensionality, they can even adjusted to be physically sound in more than two space dimensions. As a simple but suggestive application, I investigate numerically the unconventional Josephson effect in a model of a parafermion chain [19] (roughly, a system designed to emerge on the edge of an FQH liquid [20]) coupled to a Majorana chain [21, 22] by charge-conserving tunneling of spinless electrons. In one dimension, and in the continuum, these systems can also be described with the aid of bosonization [23]. However, unlike the formalism of this paper, bosonization does not grant direct numerical access to the problem considered here.

The direct link between the formalism of this paper and traditional approaches to the FQH effect has yet to be established. There is however a potentially significant indirect connection already in place. The fractionalized fermions of this paper directly linked to parafermions, and parafermions have in turn been recently linked to the FQH effect [24-26]. The relationship can be suspected from the fact that parafermions fractionalize Majorana modes [20], while in this paper the very fermions are fractionalized by comparable algebraic means. A second-quantized description of parafermions was introduced in Ref. [27], but the 
relationship of that formalism to fermion fractionalization was not recognized at the time. Our main focus here is on describing a more general, direct approach to modeling by creation and annihilation operators a fraction of a fermion carrying a non-Abelian charge like spin, in a way consistent with a lattice regulator and not restricted to one dimension.

\section{FERMION-ROOT QUASIPARTICLES}

Imagine a charge-conserving breakup/recombination process $e \leftrightarrow m e^{*}$ in which a fermion of charge $e$ decays into $m$ indistinguishable quasiparticles of charge $e^{*}=e / m$, or $m$ quasiparticles recombine into a fermion. The integer $m=2,3, \ldots$ is the number of quasiparticles that are produced when the fermion undergoes charge breakup because it left a normal medium and entered a fractionalizing one, for example. How should one effectively describe this process, in second quantization and on a lattice? Because of the lattice regularization, the process $e \leftrightarrow m e^{*}$ may be modeled as taking place at one site, or more precisely, at one single-particle state $\alpha$. Then $e \leftrightarrow m e^{*}$ translates into the equations

$$
C_{\alpha}^{\dagger m}=f_{\alpha}^{\dagger}, \quad C_{\alpha}^{m}=f_{\alpha} .
$$

These equations state that creating or annihilating $m$ quasiparticles in the state $\alpha$ is the same thing as creating or annihilating a fermion in that state. Thus, the task is to find quasiparticle operators $C_{\alpha}, C_{\alpha}^{\dagger}$ such that a) their $m$ th powers satisfy canonical anticommutation relations, and b) they have a second quantization interpretation of their own. The quasiparticles they create or annihilate are the fermion-root quasiparticles of this paper.

It is possible to split the task of solving Eq. (1) into two steps. Let us examine first the case when there is only one single-particle state, that is, only one label $\alpha$. Then one need not worry about exchange statistics. Only exclusion statistics [28] and the more technical problem of normal ordering matter. The exclusion statistics of the fermion-root quasiparticles follows from Eq. (1), since then

$$
C_{\alpha}^{\dagger 2 m}=\left(C_{\alpha}^{\dagger m}\right)^{2}=f_{\alpha}^{\dagger 2}=0, \quad C_{\alpha}^{2 m}=\left(C_{\alpha}^{m}\right)^{2}=f_{\alpha}^{2}=0
$$

This means that the state $\alpha$ can be occupied by at most by $2 m-1$ quasiparticles. It is also necessary to be able to normal-order quasiparticle creation and annihilation operators. The rule for exchanging creation and annihilation operators is

$$
C_{\alpha}^{l} C_{\alpha}^{\dagger l}+C_{\alpha}^{\dagger(2 m-l)} C_{\alpha}^{2 m-l}=\mathbb{1}, \quad l=1, \ldots, 2 m-1 .
$$


Then, as a consequence, the associated "composite" fermion satisfies the canonical relation

$$
C_{\alpha}^{m} C_{\alpha}^{\dagger m}+C_{\alpha}^{\dagger m} C_{\alpha}^{m}=f_{\alpha} f_{\alpha}^{\dagger}+f_{\alpha}^{\dagger} f_{\alpha}=\mathbb{1} .
$$

There is a simple interpretation of Eq. (3). The operator $C_{\alpha}^{\dagger(2 m-l)} C_{\alpha}^{2 m-l}$ annihilates any state with less than $2 m-l$ particles, and $C_{\alpha}^{l} C_{\alpha}^{\dagger l}$ annihilates any state with $2 m-l$ or more particles. Then Eq. (3), the rule for normal ordering quasiparticles and quasiholes, states that these two sets of states taken together conform the totality of the Fock space (since there is only one label $\alpha$ ). This interpretation also suggests that

$$
N_{\alpha}=\sum_{l=1}^{2 m-1} C_{\alpha}^{\dagger l} C_{\alpha}^{l}
$$

might play the role of number operator for the quasiparticles. To check that this is correct, one exploits the normal ordering and exclusion rules to show that

$$
\left[N_{\alpha}, C_{\alpha}^{l}\right]=-l C_{\alpha}^{l}, \quad l=1, \ldots, 2 m-1
$$

In other words, $N_{\alpha}$ generates $U(1)$ rotations of the quasiparticle creation and annihilation operators. As a consequence, fermion-root quasiparticles can be counted and minimally coupled to gauge fields. Moreover, the Fock vacuum $|0\rangle$ for the quasiparticles is defined by the usual condition $C_{\alpha}|0\rangle=0$, since then $N_{\alpha}|0\rangle=0$. The full quasiparticle Fock space is spanned by the states $|l\rangle=C_{\alpha}^{\dagger l}|0\rangle$ with $0 \geq l<2 m$ quasiparticles.

Our discussion so far solves completely the problem of taking the $m$ th root of a single fermion. The solution displays nice physical properties. If there is more than one singleparticle state, then one must in addition determine the exchange statistics of the fermion-root quasiparticles. At this point it becomes necessary to order the single particle labels, so let us index them with an integer $i$. Let $C_{\alpha_{i}}^{\prime}$ denote a set of operators satisfying the exclusion and normal ordering rules, Eqs. (2) and (3), and commuting if they carry different labels. The associated number operators are $N_{\alpha_{i}}^{\prime}$. One can construct such a set by tensoring the $2 m \times 2 m$ matrices

$$
C=\left(\begin{array}{ccccc}
0 & 1 & 0 & \cdots & 0 \\
0 & 0 & 1 & \cdots & 0 \\
0 & 0 & 0 & \cdots & 0 \\
\vdots & \vdots & \vdots & & \vdots \\
0 & 0 & 0 & \cdots & 1 \\
0 & 0 & 0 & \cdots & 0
\end{array}\right), \quad N=\left(\begin{array}{ccccc}
0 & 0 & 0 & \cdots & 0 \\
0 & 1 & 0 & \cdots & 0 \\
0 & 0 & 2 & \cdots & 0 \\
\vdots & \vdots & \vdots & & \vdots \\
0 & 0 & 0 & \cdots & 0 \\
0 & 0 & 0 & \cdots & 2 m-1
\end{array}\right)
$$


with identity matrices. Hence, if there are $N$ single-particle labels $\alpha_{i}$, then the dimension of the underlying Hilbert space is $(2 m)^{N}$. With these definitions, the quasiparticle creation and annihilation operators

$$
C_{\alpha_{i}}=C_{\alpha_{i}}^{\prime} e^{i \theta \sum_{j<i} N_{\alpha_{j}}^{\prime}}, \quad C_{\alpha_{i}}^{\dagger}=\left(C_{\alpha_{i}}^{\prime}\right)^{\dagger} e^{-\mathrm{i} \theta \sum_{j<i} N_{\alpha_{j}}^{\prime}}
$$

satisfy, thanks to Eq. (5), the exchange rules

$$
C_{\alpha_{i}} C_{\alpha_{j}}=e^{\mathrm{i} \theta} C_{\alpha_{j}} C_{\alpha_{i}}, \quad C_{\alpha_{i}} C_{\alpha_{j}}^{\dagger}=e^{-\mathrm{i} \theta} C_{\alpha_{j}}^{\dagger} C_{\alpha_{i}}, \quad i<j
$$

By construction, the statistical angle $\theta$ for the exchange of two quasiholes (or quasiparticles) turns out to be the negative of that associated to the exchange of a quasihole with a quasiparticle. For the Laughlin series $\nu=1 / q$ of FQH liquids, the same result is obtained in a very different way from computing a Berry phase in the manifold of Laughlin's wave functions $[16,29]$. Because in this paper quasiparticles are constructed so as to yield the $m$ th root of a fermion, $\theta$ must be such that

$$
\begin{aligned}
& C_{\alpha_{i}}^{m} C_{\alpha_{j}}^{m}+C_{\alpha_{j}}^{m} C_{\alpha_{i}}^{m}=f_{\alpha_{i}} f_{\alpha_{j}}+f_{\alpha_{j}} f_{\alpha_{i}}=0, \\
& C_{\alpha_{i}}^{m} C_{\alpha_{j}}^{\dagger m}+C_{\alpha_{j}}^{\dagger m} C_{\alpha_{i}}^{m}=f_{\alpha_{i}} f_{\alpha_{j}}^{\dagger}+f_{\alpha_{j}}^{\dagger} f_{\alpha_{i}}=0, \quad i \neq j .
\end{aligned}
$$

According to Eq. (6), these conditions are equivalent to the single constraint $e^{\mathrm{i} \theta m^{2}}=-1$ on the statistical angle. There are $m^{2}$ solutions

$$
\theta_{\ell}=\frac{\pi(2 \ell+1)}{m^{2}}, \quad \ell=0,1, \ldots, m^{2}-1
$$

The various allowed values of $\theta$ are qualified by physical considerations. At the most basic level, the crucial difference between $m$ even and $m$ odd is that if $m$ is odd, and only in this case, then there exists one unconventional Fock algebra with exchange statistics $e^{i \theta_{\ell}}=-1$, for $\ell=\left(m^{2}-1\right) / 2$. For lattices of dimension $D \geq 3$, only $\theta=\pi$ is allowed by locality [30], implying that only $m$ odd is physically useful within the framework of this paper. From the point of view of Jain's composite fermion theory [13] of the FQH liquids at odd denominators, it is reassuring that $\theta=\pi$ is always allowed if $m$ is odd. Composite fermions are, first and foremost, fermions whose charge has been renormalized non-adiabatically to a fractional value. It is this non-adiabatic renormalization of the charge that is unique to two dimensions [17]. In any case, unlike the exchange angle, the exclusion and normal ordering rules are indifferent to the dimension of the lattice and whether $m$ is even or odd. 
Next I will focus on low $(D=1,2)$ dimensional lattices, in order to allow for various possible statistical angles. It is convenient to decompose $m$ as $m=2^{r} q$, with $q$ odd. For simplicity, I will discuss first the case $r=0$ and show how to adjust $\theta$ in accordance to the quasiparticles of the FQH liquids at odd-denominator filling fractions $\nu=p / q=p / m$. In the next two paragraphs, $q$ will take the place of $m$ in order to facilitate contact with the literature.

$m=q$ odd. - For general $p$ (including $p=1$ ), Berry phase calculations $[16,29]$ determine the statistical angle associated to the exchange of clusters of $p$ elementary quasiholes (or quasiparticles) to be $\theta^{\prime}=\pi p / q$. I translate this result obtained in first quantization into the exchange relations

$$
C_{\alpha_{i}}^{p} C_{\alpha_{j}}^{p}=e^{i \theta^{\prime}} C_{\alpha_{j}}^{p} C_{\alpha_{i}}^{p}=e^{\mathrm{i} \pi p / q} C_{\alpha_{j}}^{p} C_{\alpha_{i}}^{p}, \quad i<j,
$$

for clusters of $p$ quasiholes. These relations are equivalent to the additional constraint

$$
e^{i \theta p^{2}}=e^{i \theta^{\prime}}=e^{i \pi p / q}
$$

on the fundamental statistical angle $\theta$ of the fermion-root quasiparticles. As first noticed in Ref. [16] in the first-quantized framework of trial wave functions and Berry phase calculations, $\theta$ is uniquely determined by Eq. (7) and the more fundamental constraint $e^{i \theta q^{2}}=-1$. This is the procedure for computing the unique statistical angle that satisfies both exchange constraints: find any pair of integers $n_{1}, n_{2}$ with $n_{1} p+n_{2} q=1$; then,

$$
\theta_{p / q}=\frac{\pi\left(n_{1}^{2} q+n_{2}^{2} p\right)}{q} \quad(\bmod 2 \pi)
$$

There is an interesting special feature of the statistical angle $\theta=\pi / q$ associated to the Laughlin fractions $\nu=1 / q$ : In this case, the "particle-hole" combinations

$$
\Gamma_{\alpha_{i}}=C_{\alpha_{i}}+C_{\alpha_{i}}^{\dagger(2 q-1)}, \quad \Delta_{\alpha_{i}}=C_{\alpha_{i}} e^{\mathrm{i} \frac{\pi}{q} N_{\alpha}}+C_{\alpha_{i}}^{\dagger(2 q-1)}
$$

have well defined exchange statistics as well. In fact, as shown in Ref. [27], the $\Gamma_{\alpha_{i}}, \Delta_{\alpha_{i}}$ satisfy the algebra of $\mathbb{Z}_{2 q}$ parafermions [31-33]. For $q=1$, that is, when there is no fractionalization, the unconventional Fock algebras of this paper reduce to the usual fermionic algebra, and Eq. (8) yields Majorana fermions

$$
\Gamma_{\alpha_{i}}=C_{\alpha_{i}}+C_{\alpha_{i}}^{\dagger} \equiv a_{\alpha_{i}}, \quad \Delta_{\alpha_{i}}=C_{\alpha_{i}}(-1)^{C_{\alpha_{i}}^{\dagger} C_{\alpha_{i}}}+C_{\alpha_{i}}^{\dagger}=-C_{\alpha_{i}}+C_{\alpha_{i}}^{\dagger} \equiv-\mathrm{i} b_{\alpha_{i}}
$$


This connection between parafermions and Majorana fermions is important because it permits to extend the ideas of Ref. [21] in order to build a toy model of a one-dimensional fractional topological superconductor. We will come back to this point in the next section.

$m$ even. - The tight link between charge and anyon statistics is lost if $m=2^{r} q$ is even [16]. This is not to say that one cannot take an even root of a fermion. But there seems to be no physical grounds to prefer any one unconventional Fock algebra when $m$ is even. We will make one more comment on this point below.

The last step in the complete determination of fermion-root quasiparticles is to confront the question, what is the exchange statistics between fractionalized and non-fractionalized fermions? Suppose the system of fractionalized electrons $f_{\alpha_{i}}=C_{\alpha_{i}}^{m}$ coexists with unfractionalized fermions $c_{\beta_{j}}$. The creation and annihilation operators $c_{\beta_{j}}^{\dagger}, c_{\beta_{j}}$ create/annihilate ordinary, unfractionalized fermions in the single-particle states $\beta_{j}$. Let us order the totality of the labels $\alpha_{i}, \beta_{j}$ so that $\alpha$-labels precede the $\beta$-labels. Because of locality (we will see a concrete instance in the next section), fermions composite or elementary must anticommute,

$$
f_{\alpha_{i}} c_{\beta_{j}}+c_{\beta_{j}} f_{\alpha_{i}}=C_{\alpha_{i}}^{m} c_{\beta_{j}}+c_{\alpha_{j}} C_{\beta_{i}}^{m}=0, \quad f_{\alpha_{i}} c_{\beta_{j}}^{\dagger}+c_{\alpha_{j}}^{\dagger} f_{\beta_{i}}=C_{\alpha_{i}}^{m} c_{\beta_{j}}^{\dagger}+c_{\beta_{j}}^{\dagger} C_{\alpha_{i}}^{m}=0 .
$$

This requirement introduces a new statistical angle $\psi$ in the problem such that

$$
C_{\alpha_{i}} c_{\beta_{j}}=e^{i \psi} c_{\beta_{j}} C_{\alpha_{i}}, \quad C_{\alpha_{i}} c_{\beta_{j}}^{\dagger}=e^{-i \psi} c_{\beta_{j}}^{\dagger} C_{\alpha_{i}}
$$

and $e^{i m \psi}=-1$. I will not investigate $\psi$ in detail. For odd $m$, one can just require that the fermions and the fermion-root quasiparticles anticommute,

$$
\left.C_{\alpha_{i}} c_{\beta_{j}}+c_{\beta_{j}} C_{\alpha_{i}}=0=C_{\alpha_{i}} c_{\beta_{j}}^{\dagger}+c_{\beta_{j}}^{\dagger} C_{\alpha_{i}} \quad \text { (allowed only for } m \text { odd }\right)
$$

For even $m$, there is no preferable answer. For example, for $m=2$, the new exchange angle can be taken to be $\psi=\pi / 2$ so that

$$
C_{\alpha_{i}} c_{\beta_{j}}=\mathrm{i} c_{\beta_{j}} C_{\alpha_{i}}, \quad C_{\alpha_{i}} c_{\beta_{j}}^{\dagger}=-\mathrm{i} c_{\beta_{j}}^{\dagger} C_{\alpha_{i}} \quad \text { (allowed for } m=2 \text { ). }
$$

In any case, it is easy to arrange for any of these condition to hold in a matrix realization of a mixed Fock algebra combining fractionalized and unfractionalized fermions. 


\section{HYBRIDIZATION OF MAJORANA AND PARAFERMION ZERO-ENERGY EDGE MODES BY CHARGE-CONSERVING TUNNELING}

Now the specification of the fermion-root quasiparticles is complete and so a concrete application is due. In this section I will consider a system of $N$ fractionalized spinless fermions $f_{i}=C_{i}^{m}$ hopping on a chain with sites $i=1, \ldots, N$, and $M$ unfractionalized fermions $c_{j}$ hopping on another chain with sites $j=1, \ldots, M$, see Fig. 1 . The Hamiltonian for the unfractionalized fermions is

$$
H_{K}=-t_{K} \sum_{j=1}^{M-1}\left(-\mathrm{i} b_{j}\right) a_{i+1}=-t_{K} \sum_{j=1}^{M-1}\left(-c_{i}+c_{i}^{\dagger}\right)\left(c_{i+1}+c_{i+1}^{\dagger}\right) .
$$

This is the Hamiltonian for the Majorana chain of Kitaev [21] deep in its topologically nontrivial regime, with perfectly decoupled Majorana zero-energy edge modes $a_{1}, b_{M}$. No topological number has been computed for the parafermion generalization

$$
H_{\mathrm{P}}=-t_{P} \sum_{i=1}^{N-1}\left(\Delta_{i} \Gamma_{i+1}^{\dagger}+H . c .\right)=-t_{P} \sum_{i=1}^{N-1}\left(C_{i} e^{\mathrm{i} \frac{\pi}{q} N_{\alpha}}+C_{i}^{\dagger(2 q-1)}\right)\left(C_{i+1}+C_{i+1}^{\dagger(2 q-1)}\right) .
$$

Nonetheless, $H_{\mathrm{P}}$ hosts zero-energy edge modes $\Gamma_{1}, \Delta_{N}$ that are protected simply because no relevant perturbation exists that is local in terms of quasiparticle operators [34] (see Ref. [19] for a different point of view). The full Hilbert space for both chains combined is of dimension $2^{M}(2 m)^{N}$.

Let us put the edges of the two chains in contact with tunneling Hamiltonians

$$
H_{1}=-\gamma\left(c_{M}^{\dagger} C_{1}^{m}-c_{M} C_{1}^{\dagger m}\right), \quad H_{2}=-\gamma\left(c_{1}^{\dagger} C_{N}^{m}-c_{1} C_{N}^{\dagger m}\right)
$$

They commute with each other and they commute with the total-charge operator

$$
Q_{\text {total }}=e^{*} \sum_{i=1}^{N} \sum_{l=1}^{2 m-1} C_{i}^{\dagger l} C_{i}^{l}+e \sum_{j=1}^{M} c_{j}^{\dagger} c_{j}=\frac{e}{m} \sum_{i=1}^{N} N_{i}+e \sum_{j=1}^{M} n_{j} .
$$

If the tunneling amplitude $\gamma$ for charge breakup processes does not vanish, then the edge modes of the two chains will hybridize. To investigate the edge-modes that may emerge as a consequence, let us introduce a quasiparticle hopping term

$$
H(\Phi)=-t\left(C_{M} C_{1}^{\dagger} e^{-\mathrm{i} \Phi / 2 m}+C_{1} C_{M}^{\dagger} e^{\mathrm{i} \Phi / 2 m}\right)
$$

that closes the system into a short ring junction. Like the tunneling Hamiltonians, $H(\Phi)$ commutes with the total charge operator. The phase $\Phi$, measured in units of the superconducting flux quantum $\Phi_{0}=h c / 2|e|=h c / 2 m\left|e^{*}\right|$, induces an equilibrium Josephson current 
through the ring. The total Hamiltonian of the ring junction is

$$
H_{\text {junction }}=H_{K}+H_{P}+H_{1}+H_{2}+H(\Phi) \text {. }
$$

For $m=3$, exact diagonalization of the junction Hamiltonian shows that the the period of the equilibrium Josephson current in this is $6 \pi$, see Fig. 1. Hence, the hybridization of the Majorana and $\mathbb{Z}_{6}$ parafermion edge modes generates two $\mathbb{Z}_{3}$ parafermion edge modes. But, unfortunately [26], the host system is not critical. Ref. [34] describes a way of realizing a $\mathbb{Z}_{3}$ parafermion chain on a critical line.

\section{THE $m$ TH ROOT OF A FERMION CARRYING A NON-ABELIAN CHARGE}

The principal attribute of fermion-root quasiparticles is that they carry a fraction of the electric charge of the underlying fermions. But what if the fermions carry also nonAbelian charges? Non-Abelian charges are quantized and tied to an intrinsic scale set by the commutation relations of the non-Abelian charges. They cannot be fractionalized or renormalized in the same way Abelian charges can be. This observation is perplexing because fermion-root quasiparticles carry the same single-particle labels that characterize the underlying fermions. It must be that the non-Abelian quantum numbers carried by the labels $\alpha$ are an attribute of the composite fermion $C_{\alpha}^{m}$, and not of the quasiparticle $C_{\alpha}$. So what are the non-Abelian quantum numbers carried by the fermion-root quasiparticles? Next I will provide a natural, but possibly non-unique in a physical sense, answer to this question. For concreteness I will focus on systems of spinful electrons. The generalization to other internal, compact non-Abelian symmetries is straightforward.

For spinful electrons, the single-particle labels are pairs $\boldsymbol{r}, \sigma$ consisting of a lattice site $\boldsymbol{r}$ and the spin component $\sigma=\uparrow, \downarrow$ along the quantization axis. Now imagine a spin-conserving system split into a normal (non-fractionalizing) region with Hamiltonian $H_{N}$ (lattice sites $\boldsymbol{x}$ ), a fractionalizing region $H_{F}$ (lattice sites $\boldsymbol{y}$ ), and an interface $H_{I}$. The interface Hamiltonian contains only terms of the form of Eq. (9). If I at first I take $H_{F}=0$, then the total Hamiltonian for the system is $H_{N}+H_{I}$, and the total spin operator is

$$
J^{a}=\sum_{\boldsymbol{x}} s_{\boldsymbol{x}}^{a}+\sum_{\boldsymbol{y}} S_{\boldsymbol{y}}^{a}, \quad a=x, y, z
$$


For the normal region, the local spin is given as usual by $s_{\boldsymbol{x}}^{-}=f_{\boldsymbol{x}, \downarrow}^{\dagger} f_{\boldsymbol{x}, \uparrow}, s_{\boldsymbol{x}}^{z}=\left(n_{\boldsymbol{x}, \uparrow}-n_{\boldsymbol{x}, \downarrow}\right) / 2$. For the composite fermions at the interface, the local spin is

$$
S_{\boldsymbol{y}}^{-}=C_{\boldsymbol{y}, \downarrow}^{\dagger m} C_{\boldsymbol{y}, \uparrow}^{m}, \quad S_{\boldsymbol{y}}^{z}=\frac{1}{2}\left(C_{\boldsymbol{y}, \uparrow}^{\dagger m} C_{\boldsymbol{y}, \uparrow}^{m}-C_{\boldsymbol{y}, \downarrow}^{\dagger m} C_{\boldsymbol{y}, \downarrow}^{m}\right) .
$$

Now rather than asking what is the correct $J^{a}$ for a non-trivial $H_{F}$, I propose that $J^{a}$ is always correct. Then the question is, can one build model Hamiltonians $H_{F}$ that conserve spin and display deconfined fractional charge? The answer is in the affirmative, because the total charge per site $Q_{\boldsymbol{y}}=e^{*}\left(N_{\boldsymbol{y}, \uparrow}+N_{\boldsymbol{y}, \downarrow}\right)$ commutes with the the spin operators of Eq. (12),

$$
\left[Q_{\boldsymbol{y}}, S_{\boldsymbol{y}^{\prime}}^{-}\right]=0=\left[Q_{\boldsymbol{y}}, S_{\boldsymbol{y}^{\prime}}^{z}\right]
$$

Hence, one can construct an alternative set of quasiparticles that carry fractional charge and definite values of spin. These are the elementary excitations of the fractionalizing nonAbelian medium, and the fundamental objects for building model Hamiltonians.

Let me denote by $L_{k}=\left(l_{1}, l_{2}, l_{3}, l_{4}\right)$ the set of $l_{i}=0, \ldots, 2 m-1$ such that $l_{1}-l_{2}+l_{3}-l_{4}=k$, with $k$ an integer $|k| \leq 2(2 m-1)$. Then the combinations $C_{\boldsymbol{y}}^{L_{k}}=C_{\boldsymbol{y}, \uparrow}^{\dagger l_{1}} C_{\boldsymbol{y}, \uparrow}^{l_{2}}, C_{\boldsymbol{y}, \downarrow}^{\dagger l_{3}} C_{\boldsymbol{y}, \downarrow}^{l_{4}}$ change the local amount of charge by $k e^{*}$ units,

$$
\left[Q_{\boldsymbol{y}^{\prime}}, C_{\boldsymbol{y}}^{L_{k}}\right]=k e^{*} C_{\boldsymbol{y}}^{L_{k}} \delta_{\boldsymbol{y}, \boldsymbol{y}^{\prime}}
$$

The excitations created by these combinations are not eigenstates of spin in general. But because spin commutes with the local charge, a rotation in spin space does not mix different $k$. Hence one obtains a linear transformation

$$
\left[S_{\boldsymbol{y}}^{a}, C_{\boldsymbol{y}}^{L}\right]=\sum_{L_{k}^{\prime}}\left(j^{a}\right)_{L_{k}^{\prime}}^{L_{k}} C_{\boldsymbol{y}}^{L_{k}^{\prime}} .
$$

From this point on one follows the standard theory of tensor operators. The angular momentum matrix $j^{z}$ can be diagonalized by introducing linear combinations of the $C_{\boldsymbol{r}}^{L_{k}}$. These new quasiparticles $A_{\boldsymbol{y}}^{K}, K=\left(k, s, m_{s}\right)$, carry charge $k e^{*}$, and spin $s(s+1)$ with projection $m_{s}$ onto the quantization axis. The $A$ quasiparticles have well defined exchange statistics since

$$
A_{\boldsymbol{y}_{1}}^{K_{1}} A_{\boldsymbol{y}_{2}}^{K_{2}}=e^{i \theta k_{1} k_{2}} A_{\boldsymbol{y}_{2}}^{K_{2}} A_{\boldsymbol{y}_{1}}^{K_{1}}
$$

A spin-conserving hopping term reads $A_{\boldsymbol{y}}^{K} A_{\boldsymbol{y}^{\prime}}^{K \dagger}+A_{\boldsymbol{y}^{\prime}}^{K} A_{\boldsymbol{y}}^{K \dagger}$.

The change of basis from the $C$ to $A$ quasiparticles is computationally inexpensive because it is local: all one has to do is to compute the change of basis for a system with only one 
site. Then, the dimension of the relevant Fock space is $4 m^{2}$, and the matrices $j^{a}$ are of small dimension. Moreover, they are easy to compute because the $C^{L_{k}}$ are orthogonal with respect to the trace inner product. I will not show the outcome of such a systematic calculation here. I will present one spin 1/2 multiplet of fractionally-charged quasiholes to illustrate some counterintuitive features. The $A$ quasihole

$$
A_{\boldsymbol{y}}^{\downarrow}=C_{\boldsymbol{y}, \uparrow}^{\dagger 2} C_{\boldsymbol{y}, \uparrow}^{3}-C_{\boldsymbol{y}, \uparrow}^{\dagger 3} C_{\boldsymbol{y}, \uparrow}^{4}
$$

of fractional charge $-e^{*}$ features no "spin" down $C$ quasiparticles. Nonetheless, it lowers the angular momentum along the quantization axis, since $\left[S_{\boldsymbol{y}}^{z}, A_{\boldsymbol{y}}^{\downarrow}\right]=-(1 / 2) A_{\boldsymbol{y}}^{\downarrow}$. Also, it is a member of a spin $1 / 2$ multiplet because $\left[S_{\boldsymbol{y}}^{-}, A_{\boldsymbol{y}}^{\downarrow}\right]=0$. Hence, one can compute the other member of the multiple as $A_{\boldsymbol{y}}^{\uparrow}=\left[S_{\boldsymbol{y}}^{+}, A_{\boldsymbol{y}}^{\downarrow}\right]$, which yields

$$
A_{\boldsymbol{y}}^{\uparrow}=-C_{\boldsymbol{r}, \uparrow}^{\dagger 2} C_{\boldsymbol{r}, \downarrow}^{3}+C_{\boldsymbol{r}, \uparrow}^{\dagger 3} C_{\boldsymbol{r}, \uparrow} C_{\boldsymbol{r}, \downarrow}^{3}-C_{\boldsymbol{r}, \uparrow}^{\dagger 5} C_{\boldsymbol{r}, \uparrow}^{3} C_{\boldsymbol{r}, \downarrow}^{3}
$$

and check explicitly that $\left[S_{\boldsymbol{y}}^{z}, A_{\boldsymbol{y}}^{\uparrow}\right]=(1 / 2) A_{\boldsymbol{y}}^{\uparrow}$ and $\left[S_{\boldsymbol{y}}^{+}, A_{\boldsymbol{y}}^{\uparrow}\right]=0$. The adjoint spin $1 / 2$ multiplet $A_{\boldsymbol{y}}^{\downarrow \dagger}, A_{\boldsymbol{y}}^{\uparrow \dagger}$ creates quasiparticles of charge $e^{*}$.

\section{SUMMARY AND OUTLOOK}

The procedure of taking the $m$ th root of a fermion yields $m^{2}$ unconventional Fock algebras. The associated quasiparticles satisfy $2 m$-exclusion statistics, that is, Eq. (2), and the normal ordering rule Eq. (3). These two properties suffice to enforce the key result that the number operator defined in Eq. (4) generates $U(1)$ rotations of the fermion-root quasiparticles, see Eq. (5). The only feature that distinguishes the various types of fermion-root quasiparticles is the statistical angle $\theta_{\ell}=\pi(2 \ell+1) / m^{2}, \ell=1, \ldots, m^{2}-1$. If the system under investigation features both fractionalized and unfractionalized fermions, then, in order to respect locality, one must demand that the "composite" fermions obtained from filling a single-particle state with $m$ fermion-root quasiparticles anticommute with the unfractionalized fermions. This condition introduces a new statistical angle $\psi$ in the problem for characterizing the result of exchanging an unfractionalized fermion with a fermion-root quasiparticle. There are simple concrete matrix realizations of these unconventional Fock algebras. Finally, if the fractionalized fermions carry non-Abelian charges, then then the fermion root quasiparticles do not carry well defined values of these charges. But one can make a rotation in charge 
space in order to obtain quasiparticles that carry fractional charge and well defined values of the non-Abelian charges.

I conclude with some ideas for future research. One possibility would be to model other mesoscopic(-inspired) systems like the hybrid chain of this paper. The study of mesoscopic devices that include fractionalizing components is an active field in connection to topological quantum computation [20, 24-26]. Using the quasiparticles of this paper it would be straightforward, thanks to advances in computational many-body physics like tensor networks, to study various forms of fractionalized Andreev bound states numerically [35]. Another possibility would be to study the shot noise spectrum of pinched fractional edge channels, at moderate values of the effective inter-edge transmission where the Luttinger liquid approach becomes intractable (see Ref. [36], and references therein).

\section{ACKNOWLEDGEMENTS}

I would like to gratefully acknowledge discussions with Denis Chevallier, Wellington Galleas, Bernard van Heck, Gerardo Ortiz, Dirk Schuricht, and Cristiane Morais Smith. This work is part of the DITP consortium, a program of the Netherlands Organisation for Sci-

entific Research (NWO) that is funded by the Dutch Ministry of Education, Culture and Science $(\mathrm{OCW})$.

\section{REFERENCES}

[1] K. G. Wilson, Phys. Rev. D 10, 2445 (1974).

[2] X.-G. Wen, Phys. Rev. B 65, 165113 (2002).

[3] A. Yu. Kitaev, Ann. Phys. 303, 2 (2003).

[4] Z. Nussinov and G. Ortiz, Ann. Phys. 324, 977 (2009).

[5] R. Savit, Rev. Mod. Phys. 52, 453 (1980).

[6] E. Cobanera, G. Ortiz, and Z. Nussinov, Adv. Phys. 60, 679 (2011).

[7] P. W. Anderson, Phys. Rev. 109, 1492 (1958).

[8] S. Ryu, A. Schnyder, A. Furusaki, and A. Ludwig, New J. Phys. 12, 065010 (2010) 
[9] L. Amico, R. Fazio, A. Osterloh, and V. Vedral, Rev. Mod. Phys. 80, 517 (2008).

[10] W. P. Su, J. R. Schrieffer, and A. J. Heeger, Phys. Rev. Lett. 42, 1698 (1979).

[11] A.J. Niemi and G.W. Semenoff, Phys. Rep. 135, 99 (1986).

[12] S. Das Sarma and A. Pinczuk (editors), Perspective in quantum Hall effects, John Wiley \& Sons, Inc., New York (1997).

[13] O. Heinonen (editor), Composite fermions, a unified view of the quantum Hall regime, World Scientific, Singapore (1998).

[14] G. Murthy and R. Shankar, Rev. Mod. Phys. 75, 1101 (2003).

[15] I use the qualification "elementary" in the sense of Ref. [17].

[16] W. P. Su, Phys. Rev. B 34, 1031 (1986).

[17] A. S. Goldhaber, J. Math. Phys. 44, 3607 (2003).

[18] R. de-Picciotto, M. Reznikov, M. Heiblum, V. Umansky, G. Bunin and D. Mahalu1 Nature 389, 162 (1997).

[19] P. Fendley, J. Stat. Mech. P11020 (2012).

[20] N. H. Lindner, E. Berg, G. Refael, and A. Stern, Phys. Rev. X 2, 041002 (2012);

[21] A. Y. Kitaev, Phys. Usp. 44, 131 (2001).

[22] V. Mourik, K. Zuo, S. M. Frolov, S. R. Plissard, E. P. A. M. Bakkers, and L. P. Kouwenhoven, Science 336, 1003 (2012).

[23] D. J. Clarke, J. Alicea, and K. Shtengel, Nat. Commun. 4, 1348 (2013).

[24] M. Burrello, B. van Heck, and E. Cobanera, Phys. Rev. B 87, 195422 (2013)

[25] M. Cheng, Phys. Rev. B 86, 195126 (2012); A. Vaezi, Phys. Rev. B 87, 035132 (2013); Y.-Z. You, C.-M. Jian, and X.-G. Wen, Phys. Rev. B 87, 045106 (2013); M. Barkeshli, C.-M. Jian, and X.-L. Qi, Phys. Rev. B 87, 045130 (2013).

[26] R. S. K. Mong, D. J. Clarke, J. Alicea, N. H. Lindner, P. Fendley, C. Nayak, Y. Oreg, A. Stern, E. Berg, K. Shtengel, and M. P. A. Fisher, Phys. Rev. X 4, 011036 (2014).

[27] E. Cobanera and G. Ortiz, Phys. Rev. A 89, 012328 (2014).

[28] C. D. Batista and G. Ortiz, Adv. Phys. 53, 1 (2004).

[29] D. Arovas, J. R. Schrieffer, and F. Wilczek, Phys. Rev. Lett. 53, 722 (1984).

[30] There is a modern discussion of this result by N. Read J. Math. Phys. 44, 558 (2003).

[31] F. C. Alcaraz and R. Köberle, Phys. Rev. D 24, 1562 (1981).

[32] A. Jaffe and F. L. Pedrocchi, Commun. Math. Phys. 337, 445 (2015). 
[33] J. Alicea and P. Fendley, Ann. Rev. Cond. Matt. Phys. 7, 119 (2016).

[34] E. Cobanera, J. Ulrich, and F. Hassler, Phys. Rev. B 94, 125434 (2016)

[35] C. Bena, The European Physical Journal B, 85, 196 (2012). This paper studies nonfractionalizing systems only but it would be an excellent model to follow.

[36] M. Heiblum, arXiv:0912.4868 [cond-mat.mes-hall] (2009). 

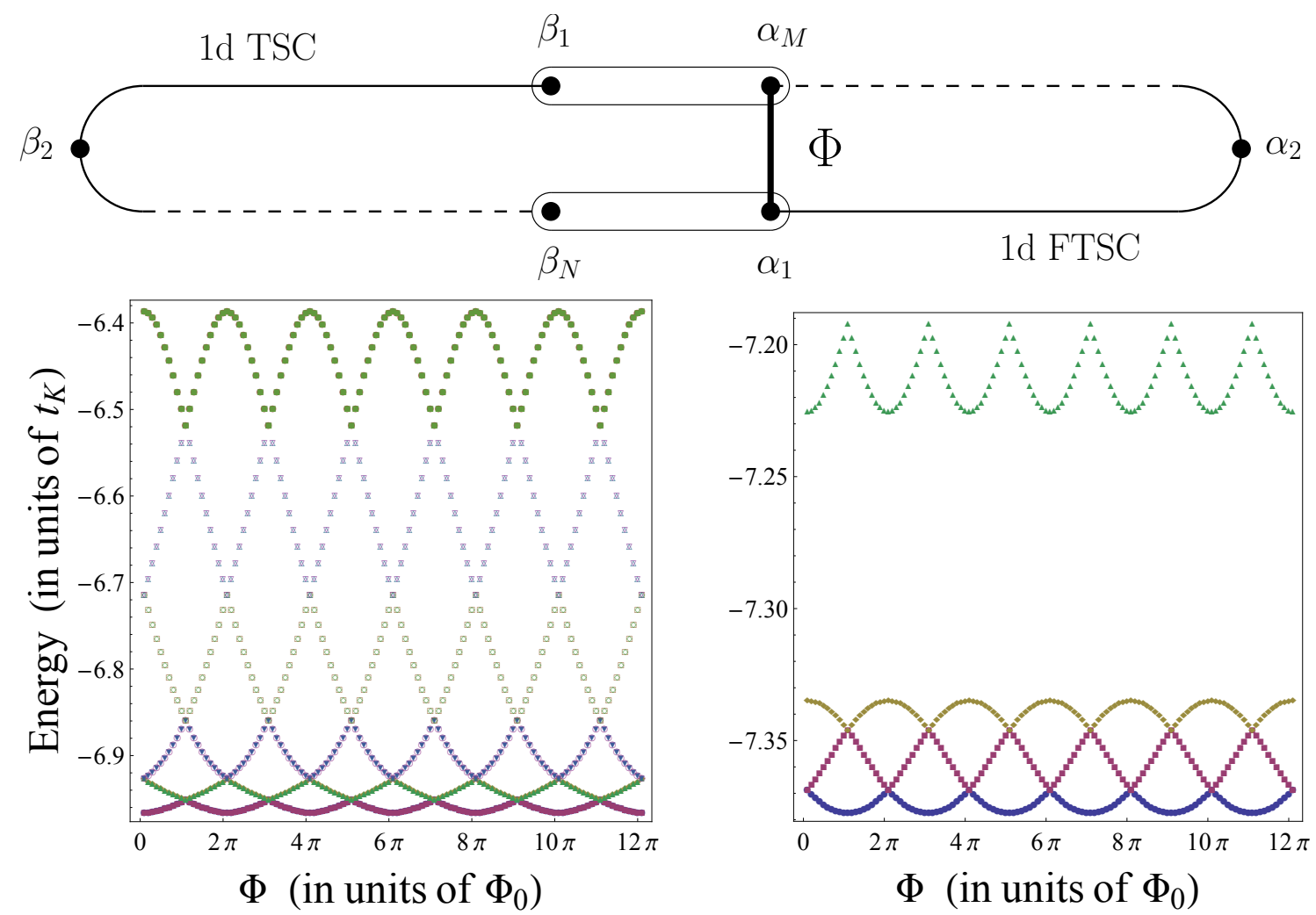

FIG. 1. Hybridization of Majorana and parafermion zero-energy edge modes. Top panel: Schematic representation of the model Hamiltonian: The endpoints of a Majorana chain $(\mathrm{D}=1$ topological superconductor (TSC)) and the endpoints of of a parafermion chain ( $\mathrm{D}=1$ fractional topological superconductor (FTSC)) are connected by fractionalizing, charge-conserving tunneling. A fractionalizing link closes the resulting hybrid wire into a ring junction, threaded by a flux $\Phi$ measured in units of $\Phi_{0}=h c / 2 e$, see Eq. (10). In the following, $m=3, t_{K}=t_{P}=t=1$, and $M=N=3$; see main text. Bottom left panel: If the chains are decoupled, exact diagonalization of the junction Hamiltonian Eq. (11) shows that the six lowest energy levels are doubly degenerate and do not cross higher levels. The period of the equilibrium supercurrent $I_{s c} \propto d E / d \Phi$ is $12 \pi$, consistent with $\mathbb{Z}_{6}$ parafermion edge modes. Bottom right panel: If the chains are coupled by tunneling, exact diagonalization shows that the three lowest energy levels of the junctions are non-degenerate and do not cross higher levels. The period of the supercurrent is $6 \pi$, consistent $\mathbb{Z}_{3}$ parafermion edge modes. 